\title{
Acknowledgment to reviewers of Journal of Surveillance, Security and Safety in 2021
}

\author{
JSSS Editorial Office \\ JSSS Editorial Office, Alhambra, CA 91801, USA. \\ Correspondence to: JSSS Editorial Office, 245 E Main Street Ste 107, Alhambra, CA 91801, USA. \\ E-mail: editorial@jsssjournal.com
}

How to cite this article: JSSS Editorial Office. Acknowledgment to reviewers of Journal of Surveillance, Security and Safety in 2021. J Surveill Secur Saf 2022;3:1-2. https://dx.doi.org/10.20517/jsss.2022.03

Received: 17 Jan 2022 Accepted: 18 Jan 2022 Published: 19 Jan 2022

Academic Editor: S. S. lyengar Copy Editor: Xi-Jun Chen Production Editor: Xi-Jun Chen

Rigorous peer-review is the corner-stone of high-quality academic publishing. The editors of Journal of Surveillance, Security and Safety (JSSS) would like to express their sincere gratitude to the following reviewers for their precious time and dedication, regardless of whether the papers were finally published in 2021 [Table 1].

\section{Table 1. The reviewers in 2021}

\begin{tabular}{|c|c|c|c|c|}
\hline \multicolumn{5}{|l|}{ Names } \\
\hline Massimo Ficco & Dohyun Kim & Kshirasagar S. Naik & Seokwon Yeom & Shaun S. Wang \\
\hline Mohammed Bouhorma & Gautam Srivastava & Denis Scalbert & Weiqing Sun & Stefanos Gritzalis \\
\hline Zheng Jun & Debiao He & David Hutchison & Dana Petcu & Yuliy Baryshnikov \\
\hline Rajesh Kumar & Wenhai Sun & Vincenzo De Florio & Allel Hadjali & Karl Andersson \\
\hline Yongxin Liu & Ken Barker & Saru Kumari & Keke Gai & Gerhard Wilhelm Weber \\
\hline Houbing Song & Sherali Zeadally & Chunwei Tsai & Dickson K.W. Chiu & Russell G. Thompson \\
\hline Rosa María Arnaldo Valdés & Nora Cuppens & Christos Aridas & Akila Siriweera & Krishna Kant \\
\hline Stathis Malakis & Michael G. Pecht & Seung Geol Choi & Yi-Hung Liu & Qi Jiang \\
\hline Rakesh Shrestha & Yashwant Malaiya & Fosca Giannotti & Christos Douligeris & Mohammad Shojafar \\
\hline Guangming Cui & Frédéric Cuppens & Unil Yun & Mohammad Hossein Anisi & Luca Calderoni \\
\hline Steven D. Galbraith & Ajita Rattani & Nathalie Japkowicz & Eirini Eleni Tsiropoulou & Massimo lanigro \\
\hline Guomin Yang & Weizhi Meng & Zhihong Tian & Goutam Chakraborty & Nan Wang \\
\hline Athanasios Vasilakos & Constantinos Patsakis & Qinghua Li & Douglas Reeves & Hui Yang \\
\hline Panagiotis Trakadas & Domenico Talia & Marc Dacier & Angela Bonifati & Felix C. Engel \\
\hline Ngoc Hong Tran & Bo Sheng & G Bianchi & Bryan Ford & Anas Husseis \\
\hline Xiaodong Lin & Krishnamurty Muralidhar & Antonio Pescapé & Qiong Huang & Jalil Nourmohammadi-Khiarak \\
\hline Chengsheng Yuan & Patrick C.K. Hung & Jemal Abawajy & Keith M. Martin & Feng-Cheng Chang \\
\hline
\end{tabular}




\section{DECLARATIONS}

\section{Authors' contributions}

Writing and revision of the article and approved the final version: JSSS Editorial Office

\section{Availability of data and materials}

Not applicable.

\section{Financial support and sponsorship}

None.

\section{Conflicts of interest}

The author declared that there are no conflicts of interest.

\section{Ethical approval and consent to participate}

Not applicable.

\section{Consent for publication}

Not applicable.

\section{Copyright}

(C) The Author(s) 2022. 